\title{
Ground-based photometry for 42 Kepler-field RR Lyrae stars
}

\author{
Young-Beom Jeon ${ }^{1}$, Chow-Choong Ngeow $^{2}$ and James M. Nemec ${ }^{3}$ \\ ${ }^{1}$ Korea Astronomy and Space Science Institute (KASI), Daejeon 305-348, Korea \\ email: ybjeon@kasi.re.kr \\ ${ }^{2}$ Graduate Institution of Astronomy, National Central University, Taiwan \\ email: cngeow@gmail.com \\ ${ }^{3}$ Department of Physics \& Astronomy, Camosun College, Victoria, British Columbia, Canada \\ email: jmn@isr.bc.ca
}

\begin{abstract}
Follow-up $(U) B V R I$ photometric observations have been carried out for $42 \mathrm{RR}$ Lyrae stars in the Kepler field. The new magnitude and color information will complement the available extensive high-precision Kepler photometry and recent spectroscopic results. The photometric observations were made with the following telescopes: 1-m and 41-cm telescopes of Lulin Observatory (Taiwan), 81-cm telescope of Tenagra Observatory (Arizona, USA), 1-m telescope at the Mt. Lemmon Optical Astronomy Observatory (LOAO, Arizona, USA), 1.8-m and 15-cm telescopes at the Bohyunsan Optical Astronomy Observatory (BOAO, Korea) and 61-cm telescope at the Sobaeksan Optical Astronomy Observatory (SOAO, Korea). The observations span from 2010 to 2013 , with $\sim 200$ to $\sim 600$ data points per light curve. Preliminary results of the Korean observations were presented at the 5th KASC workshop in Hungary. In this work, we analyze all observations. These observations permit the construction of full light curves for these RR Lyrae stars and can be used to derive multi-filter Fourier parameters.
\end{abstract}

Keywords. stars: variable: RR Lyrae

We obtained ground-based $(U) B V R I$ photometric observations for $42 \mathrm{RR}$ Lyrae stars in the Kepler field. Figure 1 shows a phased light curve of KIC 3864443 and the correlation between $\phi_{31}(K p)$ and $\phi_{31}(V)$ for all Blazhko and non-Blazhko RRab stars. Table 1 presents the total amplitudes, $A_{\text {tot }}$, and $\phi_{31}$ parameters from Fourier analysis of RRab
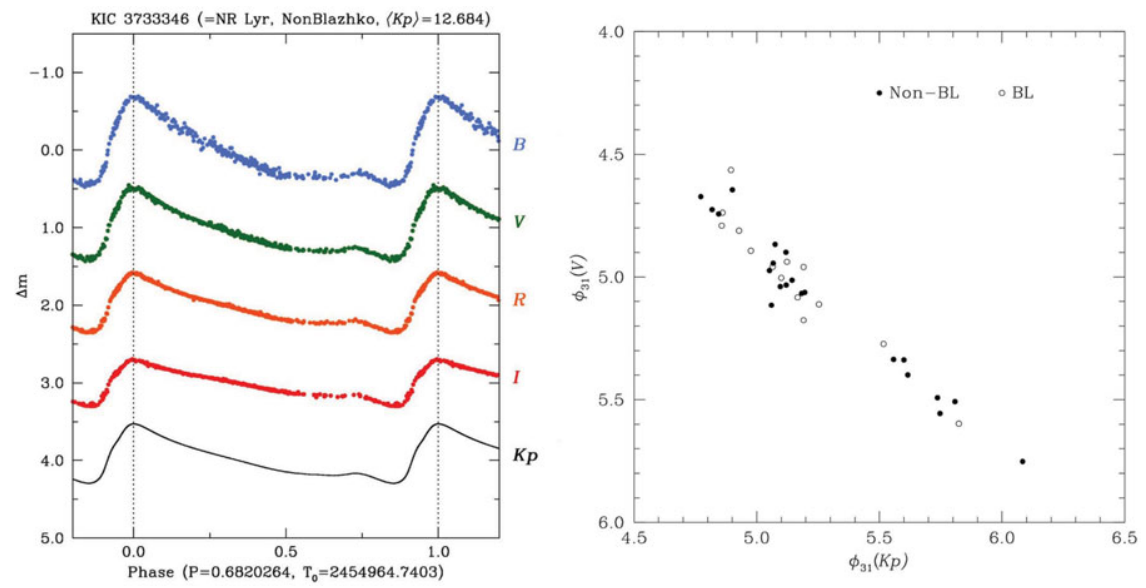

Figure 1. A sample phased light curve of KIC 3864443 (left) and the correlation between $\phi_{31}(K p)$ and $\phi_{31}(V)$ for Blazhko and non-Blazhko RRab stars (right). 
Table 1. Fourier parameters for RRab stars

\begin{tabular}{|c|c|c|c|c|c|c|c|c|c|c|}
\hline KIC & GCVS & $\begin{array}{l}\text { Period } \\
\text { day }\end{array}$ & $\begin{array}{c}\mathbf{A}_{\text {tot }}(B) \\
\text { mag }\end{array}$ & $\begin{array}{c}\phi_{31}(B) \\
\operatorname{rad}\end{array}$ & $\begin{array}{c}\mathbf{A}_{\text {tot }}(V) \\
\text { mag }\end{array}$ & $\begin{array}{c}\phi_{31}(V) \\
\operatorname{rad}\end{array}$ & $\begin{array}{c}\mathbf{A}_{\text {tot }}(R) \\
\text { mag }\end{array}$ & $\begin{array}{c}\phi_{31}(R) \\
\operatorname{rad}\end{array}$ & $\begin{array}{c}\mathbf{A}_{\text {tot }}(I) \\
\text { mag }\end{array}$ & $\begin{array}{c}\phi_{31}(I) \\
\mathrm{rad}\end{array}$ \\
\hline \multicolumn{11}{|l|}{ Blazhko: } \\
\hline 3864443 & V2178 Cyg & 0.48695 & 1.106 & 4.245 & 0.834 & 4.564 & 0.712 & 4.556 & 0.554 & 4.905 \\
\hline 4484128 & V808 Cyg & 0.54786 & 1.398 & 4.856 & 1.115 & 5.055 & 0.922 & 5.254 & 0.707 & 5.621 \\
\hline 5559631 & V783 Cyg & 0.6207 & 1.220 & 5.105 & 0.969 & 5.263 & 0.783 & 5.609 & 0.625 & 5.973 \\
\hline 6183128 & V354 Lyr & 0.56169 & 1.028 & 4.942 & 0.821 & 5.168 & 0.639 & 5.496 & 0.490 & 6.082 \\
\hline 6186029 & V 445 Lyr & 0.51312 & 0.664 & {$[6.514]$} & 0.529 & {$[6.964]$} & 0.464 & {$[7.106]$} & 0.449 & 5.030 \\
\hline 7257008 & - & 0.51178 & 1.252 & 4.632 & 0.997 & 4.769 & 0.739 & 5.149 & 0.594 & 5.057 \\
\hline 7505345 & V355 Lyr & 0.4737 & 1.417 & 4.796 & 1.189 & 4.894 & 0.965 & 4.990 & 0.756 & 5.362 \\
\hline 7671081 & V450 Lyr & 0.50461 & 1.122 & 4.519 & 0.873 & 4.919 & 0.736 & 4.884 & 0.614 & 5.381 \\
\hline 9001926 & V353 Lyr & 0.5568 & 1.288 & 4.714 & 1.004 & 4.879 & 0.830 & 5.202 & 0.699 & 5.721 \\
\hline 9578833 & V366 Lyr & 0.52703 & 1.344 & 4.935 & 1.067 & 5.090 & 0.869 & 5.385 & 0.662 & 5.576 \\
\hline 9697825 & V360 Lyr & 0.55758 & 1.005 & 4.755 & 0.760 & 4.991 & 0.669 & 5.114 & 0.479 & 5.771 \\
\hline 9973633 & - & 0.51075 & 1.506 & 5.240 & 1.315 & 5.107 & 0.909 & 5.206 & 0.732 & 5.526 \\
\hline 10789273 & V838 Cyg & 0.48028 & 1.645 & 4.645 & 1.335 & 4.784 & 1.131 & 4.933 & 0.875 & 5.236 \\
\hline 11125706 & - & 0.61322 & 0.706 & 5.302 & 0.540 & 5.614 & 0.452 & 5.970 & 0.352 & {$[6.400]$} \\
\hline 12155928 & V1104 Cyg & 0.43639 & 1.603 & 4.657 & 1.295 & 4.689 & 1.062 & 4.851 & 0.891 & 5.242 \\
\hline \multicolumn{11}{|c|}{ Non-Blazhko: } \\
\hline 3733346 & NR Lyr & 0.68203 & 1.124 & 4.829 & 0.915 & 4.977 & 0.759 & 5.172 & 0.588 & 5.590 \\
\hline 3866709 & V715 Cyg & 0.47071 & 1.525 & 4.702 & 1.226 & 4.643 & 1.015 & 4.929 & 0.841 & 5.269 \\
\hline 5299596 & V782 Cyg & 0.52364 & 0.836 & 5.168 & 0.665 & 5.449 & 0.539 & 5.811 & 0.451 & 6.170 \\
\hline 6070714 & V784 Cyg & 0.53409 & 1.055 & 5.477 & 0.830 & 5.839 & 0.616 & 6.191 & 0.482 & {$[6.435]$} \\
\hline 6100702 & - & 0.48815 & 0.926 & 5.261 & 0.714 & 5.547 & 0.569 & 5.841 & 0.440 & {$[6.357]$} \\
\hline 6763132 & NQ Lyr & 0.58779 & 1.185 & 4.811 & 0.954 & 4.958 & 0.770 & 5.105 & 0.606 & 5.628 \\
\hline 6936115 & FN Lyr & 0.5274 & 1.482 & 4.622 & 1.226 & 4.758 & 0.996 & 4.857 & 0.813 & 5.204 \\
\hline 7021124 & - & 0.62249 & 1.489 & 4.560 & 1.176 & 5.159 & 0.957 & 5.078 & 0.686 & 5.065 \\
\hline 7030715 & - & 0.68361 & 0.944 & 5.162 & 0.732 & 5.437 & 0.605 & 5.639 & 0.473 & 6.202 \\
\hline 7176080 & V349 Lyr & 0.50707 & 0.111 & 6.213 & 0.078 & {$[8.054]$} & 0.078 & {$[6.859]$} & 0.061 & {$[7.009]$} \\
\hline 7742534 & V368 Lyr & 0.45649 & 1.666 & 4.551 & 1.327 & 4.705 & 1.104 & 4.932 & 0.976 & 5.174 \\
\hline 7988343 & V1510 Cyg & 0.58114 & 1.496 & 4.850 & 1.247 & 4.963 & 0.970 & 5.122 & 0.759 & 5.427 \\
\hline 8344381 & V346 Lyr & 0.57683 & 1.430 & 4.858 & 1.163 & 5.009 & 0.945 & 5.036 & 0.563 & 4.416 \\
\hline 9508655 & V350 Lyr & 0.59424 & 1.452 & 4.809 & 1.189 & 4.955 & 0.974 & 5.130 & 0.758 & 5.523 \\
\hline 9591503 & V894 Cyg & 0.57139 & 1.577 & 4.871 & 1.257 & 4.961 & 1.037 & 5.148 & 0.824 & 5.452 \\
\hline 9658012 & - & 0.53321 & 1.402 & 4.811 & 1.125 & 5.012 & 0.943 & 5.103 & 0.719 & 5.489 \\
\hline 9717032 & - & 0.55691 & 1.384 & 4.852 & 1.021 & 5.070 & 0.852 & 5.399 & 0.704 & 5.432 \\
\hline 9947026 & V2470 Cyg & 0.54859 & 0.880 & 5.254 & 0.693 & 5.488 & 0.571 & 5.900 & 0.458 & {$[6.344]$} \\
\hline 10136240 & V1107 Cyg & 0.56578 & 1.178 & 4.899 & 0.961 & 5.113 & 0.785 & 5.326 & 0.618 & 5.648 \\
\hline 10136603 & V1107 Cyg & 0.43377 & 1.235 & 5.186 & 0.947 & 5.121 & 0.732 & 5.667 & 0.572 & 6.139 \\
\hline 11802860 & AW Dra & 0.68722 & 1.321 & 5.240 & 1.045 & 5.431 & 0.848 & 5.639 & 0.652 & 6.045 \\
\hline
\end{tabular}

Notes: [ ] denotes $\phi_{31}$ larger than $2 \pi$.

stars. RRc stars are expected to behave differently from the RRab stars and require special treatment, so they are not included. We used IRAF/digiphot/phot program to obtain the photometry. The periods in Table 1 , as well as $T_{0}$ and $K p$ in Fig. 1, were taken from Nemec et al. (2011, 2013). The Fourier analysis was based on Géza Kovács' Fourier decomposition program.

From Table 1 we can calculate the mean differences between $\phi_{31}$ in $B V R I$ bands and $\phi_{31}(K p)$ and their standard deviations. They are the following: $\Delta \phi_{31}(B)=\left\langle\phi_{31}(B)-\right.$ $\left.\phi_{31}(K p)\right\rangle=0.430 \pm 0.107 \mathrm{rad}$ from 34 stars, $\Delta \phi_{31}(V)=0.174 \pm 0.085 \mathrm{rad}$ from 34 stars, $\Delta \phi_{31}(R)=-0.018 \pm 0.053 \mathrm{rad}$ from 34 stars and $\Delta \phi_{31}(I)=-0.192 \pm 0.063 \mathrm{rad}$ from 35 stars. Earlier, $\Delta \phi_{31}(V)=0.151 \mathrm{rad}$ was derived by Nemec et al. (2011) based on only three RR Lyrae stars. These results can help to derive metal abundances using Fourier parameters if $\phi_{31}(B, V, R, I)$ vs. $\phi_{31}(K p)$ relations will be used to translate the former to the latter and then the $[\mathrm{Fe} / \mathrm{H}]$ vs. $\phi_{31}(K p)$ relation of Nemec et al. $(2013)$ will be used to derive $[\mathrm{Fe} / \mathrm{H}]$.

Transformation to standard photometry is currently in progress.

\section{References}

Nemec, J. M., Smolec, R., Benkő, J. M., et al. 2011, MNRAS, 417, 1022

Nemec, J. M., Cohen, J. G., Ripepi, V., et al. 2013, ApJ, 773, 181 